\title{
Performance and Industrial Application of New-Type Sulfur Tolerant CO Shift Catalyst QDB-04
}

\author{
Bonan Liu ${ }^{1}$, Tiancun Xiao ${ }^{1}$, Peter P. Edwards ${ }^{1}$, Jiefei Xiao ${ }^{2 *}$, Gao Hui ${ }^{2}$, Qiuyun Zong ${ }^{*}$ \\ ${ }^{1}$ Inorganic Chemistry Laboratory, Oxford University, Oxford, UK \\ ${ }^{2}$ Qindao Lianxin Catalytic Materials Co., Ltd, Qingdao, China \\ Email: "xiao.tiancun@chem.ox.ac.uk, "xxxff@126.com, "zqy1959@163.com
}

Received 29 October 2015; accepted 3 January 2016; published 6 January 2016

Copyright (C) 2016 by authors and Scientific Research Publishing Inc.

This work is licensed under the Creative Commons Attribution International License (CC BY). http://creativecommons.org/licenses/by/4.0/

(c) (i) Open Access

\begin{abstract}
This paper presents the performance and characteristics of new-type sulfur tolerant shift catalyst QDB-04 and its industrial side-line test as well as the first-time industrial application in Lunan Chemical Fertilizer Plant of Shandong in China. The results show that the catalyst has high strength and strength stability, good low temperature activity and stability as well as low potassium bleeding ratio which well meet for the requirements of the methanol plant on catalyst performance in Lunan Chemical Fertilizer Plant.
\end{abstract}

\section{Keywords}

Sulfur Tolerant Shift Catalyst, Performance, Industrial Side-Line Test, Application

\section{Introduction}

Great attentions have been paid to the sulfur tolerant shift catalyst because of its excellent shift activity, sulfur tolerant ability and poisoning resistance. Many brands of such catalyst have been developed in the world, for example, K8-11 of BASF in Germany, SSK of TOPSOE in Denmark and QDB series of Qindao Lianxin Catalytic Materials Co., Ltd [1] [2]. The above catalysts have two kinds of supports, $\mathrm{MgAl}_{2} \mathrm{O}_{4}$ and $\gamma-\mathrm{Al}_{2} \mathrm{O}_{3} \cdot \mathrm{MgAl}_{2} \mathrm{O}_{4^{-}}$ based catalyst has better strength and stability which can be applied in large-scale ammonia plant with high pressure, high water/gas ratio [3] [4]. But these kinds of catalysts have complicated production process and high price. So the $\gamma-\mathrm{Al}_{2} \mathrm{O}_{3}$-based catalyst is the first choice of small and medium-scale chemical fertilizer plant in China. The catalyst has simple production process, low price, high low-temperature activity, but its strength and

\footnotetext{
"Corresponding authors.
}

How to cite this paper: Liu, B.N., Xiao, T.C., Edwards, P.P., Xiao, J.F., Hui, G. and Zong, Q.Y. (2016) Performance and Industrial Application of New-Type Sulfur Tolerant CO Shift Catalyst QDB-04. Open Journal of Inorganic Chemistry, 6, 15-22. 
strength stability are not good enough, especially when it gets powdered in operation which can cause the rise of bed resistance and affect the normal operation of the production. On the other hand, this kind of catalyst has other shortcomings such as high potassium bleeding rate, worse low sulfur resistance and easy deactivation caused by devulcanization [5].

In order to solve the above problems, we adopted a new catalyst production method and developed a newtype sulfur tolerant shift catalyst QDB-04 with special inorganic and organic assistants, using a special method of potassium fixation. The catalyst was first applied in the new industrial methanol device of Shandong Lunan Second Nitrogen Fertilizer Plant of China in June 2004. In December 2005, the catalyst passed the industrial application appraisal organized by Shandong Science and Technology Committee. The catalyst has excellent strength, strength stability and activity stability, good low-temperature activity, strong resistance to low water/gas ratio and low sulfur as well as low alkaline metal bleeding ratio which well satisfy the demand of sulphur containing feed gas and medium pressure shift process conditions on catalyst performance and its comprehensive performance reaches first-rate level in China.

This paper presents the performance, characteristics and first-time industrial application of the new-type sulfur tolerant shift catalyst QDB-04 in Lunan Chemical Fertilizer Plant of Shandong in China.

\section{Methodology}

\subsection{Catalyst Preparation}

Industrial catalysts of QDB-04 with special assistants were prepared by the dry-mixed method. The catalysts were extruded with special binder in order to get excellent strength performance.

\subsection{Catalyst Activity Measurement}

The intrinsic activity of the catalyst was tested in a atmospheric micro reactor chromatography evaluation apparatus, using $\mathrm{CO}$ conversion ratio (XCO, \%) as catalytic activity expression. The composition of the feed gas was $44 \%$ CO, 25\% CO, $0.005 \%-0.05 \% \mathrm{H} 2 \mathrm{~S}$ and remaining for $\mathrm{H}_{2}$. The feed dry gas space velocity was $10,000 \mathrm{~h}^{-1}$, water-gas ratio was 1.0 , weight of samples was 0.3 grams, particle size of catalyst was from 0.25 to 0.35 millimeter. The reaction temperature was selected for $260^{\circ} \mathrm{C}, 350^{\circ} \mathrm{C}, 400^{\circ} \mathrm{C}$.

\subsection{Catalyst Characterization}

The active component of cobalt and molybdenum was determined by spectrophotometric method, and the content of potassium assistant was tested by flame photometric detector.

The strength was tested in the DL-II intelligent strength test instrument with average size as the evaluation means. The strength stability and potassium bleeding ratio were checked by hydro-thermal treatment experiment in the original size pressure evaluation device, the strength and potassium content of samples were determined after treatment for ten hours in atmosphere of nitrogen and stream with a pressure of 50 bar, a temperature of $500^{\circ} \mathrm{C}$, a space velocity of $2000 \mathrm{~h}^{-1}$ and a water-gas ratio of 1.4.

The XRD characterization was performed on the Rigaku D/Max - 1200 X-ray diffractometer, using Cu-Ka1 target, graphite monochromator, $50 \mathrm{kv}$ voltage in X-ray tube, 100 Ma current. The specific surface area, pore structure were determined by the PM-60 mercury intrusion porosimeter.

\section{Results and Discussion}

\subsection{Main Performance and Characteristics}

\subsubsection{Strength and Strength Stability}

The experiment data are shown in Table 1. The QDB-04 had excellent strength and strength stability with special production process for its support. Whether fresh sample or the ones applied in industrial plant, the strength retention ratio of QDB-04 keeps well above 90\% which shows very good strength stability.

\subsubsection{Ability of Low Sulfur Tolerance}

The ways of potassium assistant make a big influence on activity of sulfur tolerant shift catalysts [6]. With a special potassium fixation style, the "sulfur capture" ability of the catalyst can be improved by enhancing the 
Table 1. Comparison of catalyst strength and stability.

\begin{tabular}{ccccc}
\hline \multirow{2}{*}{ Item } & \multicolumn{2}{c}{ QDB-04 } & \multicolumn{2}{c}{ Industrial catalyst A } \\
\cline { 2 - 5 } & Strength, N/cm & Retention ratio & Strength N/(particle) & Retention ratio \\
\hline Fresh catalyst & 136.8 & $/$ & 78.6 & $/$ \\
Catalyst after 1 year industrial used & 131.9 & $96.4 \%$ & 48.2 & $61.3 \%$ \\
\hline
\end{tabular}

sulfur on the surface of the catalyst, lowers the sulfur loss rate of the catalyst when the $\mathrm{H}_{2} \mathrm{~S}$ concentration is low in the feed gas [7] [8], and keeps relatively more activity center, so the catalyst activity is improved under low $\mathrm{H}_{2} \mathrm{~S}$ conditions, i.e., the catalyst low sulfur tolerance is improved. The results are shown in Table 2.

Table 2 shows that when the $\mathrm{H}_{2} \mathrm{~S}$ content in the feed gas is above $0.05 \%$, the two samples have almost the same catalytic activity; but with the lowering of the $\mathrm{H}_{2} \mathrm{~S}$ content, the sample with special potassium fixation style has obviously higher catalytic activity. This shows that the sample with special potassium fixation style has better low sulfur tolerance and higher activity under low $\mathrm{H}_{2} \mathrm{~S}$ conditions.

\subsubsection{Potassium Bleeding Ratio}

After hydro thermal treatment, different samples show different potassium bleeding ration. By a special potassium fixation style, the potassium-bleeding ratio of the catalyst under the same appraisal conditions can be decreased greatly with a result of high activity stability. The experiments show in Table 3.

\subsubsection{Activity and Sulfurization Characterization}

Table 4 shows the activity test result. It can be indicated that the special assistant helps the sample get better eigen activity under high sulfur and low sulfur conditions than the samples with no such special assistant. And the most likely reason from previous research is that special assistant promotes the reduction of $\mathrm{Mo}^{6+}$ with no activity to the active $\mathrm{Mo}^{4+}$ and improves the catalyst shift activity [9].

Table 5 shows the sulfurization performance of special assistant-promoted catalyst. Its sulfurization time has been shorted obviously. There was a theory from P Hou [9] may be as the explanation which is that the added special assistant changes the integration form between the active component and support which promotes the interchange reaction of S-O bond at low temperature.

\subsection{Industrial Side-Line Test}

On the whole low temperature shift industrial device of Shandong Lunan First Nitrogen Fertilizer Plant, $600 \mathrm{~h}$ and $1500 \mathrm{~h}$ industrial side-line test were carried out under the relative process conditions to the feed gas before and after deoxidation. The running data are shown in Table 6 and Table 7.

It can be seen in Table 6 and Table 7 that:

1) Whether with oxygen-containing or deoxidized feed gas, QDB-04 always showed high shift activity; under running conditions similar to those of Lunan First Nitrogen Fertilizer Plant, the outlet CO of QDB-04 was 1.93 3.91 (for deoxidized feed gas) and 1.98 - 4.54 (for oxygen-containing feed gas) which was the process index of $\mathrm{CO}<8 \%$ of the plant at the same period of time and showed that the catalyst activity can completely satisfy the process condition requirements of the same kind of plants.

2) In the course of the test, several times of startups and shutdown occurred due to the valve failure and pipe corrosion, but the catalytic activity and bed resistance drop remained almost the same which showed that the catalyst has quite good activity and strength stability.

\subsection{Industrial Application Test}

\subsubsection{Industrial Operation Data}

The domestically designed 100,000 t/a methanol device of Shandong Lunan Second Nitrogen Fertilizer Plant adjusts its shift furnace outlet $\mathrm{CO}$ index by adjusting the water/gas ratio. Its characteristics require the catalyst selected should have excellent ability to be tolerant of low water/gas ratio $(0.3-0.4)$, relatively low activation temperature $\left(240^{\circ} \mathrm{C}\right)$ and good heat tolerance (heating point temperature $440^{\circ} \mathrm{C}$ ). For its first time operation, they chose our new-type sulfur tolerant shift catalyst QDB-04 (the diameter of the methanol shift furnace was $2.39 \mathrm{~m}$, 
Table 2. Relations between $\mathrm{H}_{2} \mathrm{~S}$ content in the feed gas and catalyst activity with different potassium fixation style.

\begin{tabular}{|c|c|c|c|c|c|c|}
\hline & $\mathrm{H}_{2} \mathrm{~S}$ content in feed gas, $\%$ & 0.05 & 0.03 & 0.02 & 0.01 & 0.005 \\
\hline \multirow{2}{*}{ CO shift ratio \% } & Sample with special potassium fixation & 48.6 & 48.0 & 47.6 & 46.0 & 46.3 \\
\hline & Sample with common potassium fixation & 48.2 & 41.2 & 37.8 & 35.2 & 32.4 \\
\hline
\end{tabular}

Table 3. Potassium content and bleeding ratio of catalysts.

\begin{tabular}{cccc}
\hline Sample & Fresh catalyst $\mathrm{K}_{2} \mathrm{O} \%$ & Hydrothermal treatment, $\mathrm{K}_{2} \mathrm{O} \%$ & Potassium bleeding raito, \% \\
\hline Special potassium fixation style & 8.57 & 6.72 & 21.59 \\
Common potassium fixation style & 8.58 & 3.26 & 62.00 \\
\hline
\end{tabular}

Table 4. Comparison of the catalyst activity under atmospheric pressure.

\begin{tabular}{ccccc}
\hline & \multicolumn{4}{c}{ CO shift ratio, \% } \\
\cline { 2 - 5 } Catalyst & $265^{\circ} \mathrm{C}$ & $350^{\circ} \mathrm{C}$ & $400^{\circ} \mathrm{C}$ \\
\cline { 2 - 5 } & $\mathrm{H}_{2} \mathrm{~S}>500 \mathrm{ppm}$ & $\mathrm{H}_{2} \mathrm{~S}>500 \mathrm{ppm}$ & $\mathrm{H}_{2} \mathrm{~S}>500 \mathrm{ppm}$ & $\mathrm{H}_{2} \mathrm{~S}, 160 \mathrm{ppm}$ \\
\hline Sample with special assistant & 7.10 & 38.64 & 59.68 & 56.13 \\
Sample without special assistant & 6.32 & 33.38 & 54.51 & 48.98 \\
\hline
\end{tabular}

Table 5. Comparison of catalyst sulfurization performance.

\begin{tabular}{ccc}
\hline Catalyst & \multicolumn{2}{c}{$\mathrm{H}_{2} \mathrm{~S}$ in the tail gas } \\
\cline { 2 - 3 } Sample with special assistant & Penetration time, $\mathrm{B}$ & 12 \\
Sample without special assistant & 4 & 27 \\
\hline
\end{tabular}

Table 6. Test data of the $1500 \mathrm{~h}$ industrial side-line test with deoxidized feed gas.

\begin{tabular}{ccccccc}
\hline \multirow{2}{*}{ Time } & Space velocity, $\mathrm{h}^{-1}$ & \multicolumn{3}{c}{ Temperature, ${ }^{\circ} \mathrm{C}$} & Tail gas, CO\% & Shift ratio, \% \\
\cline { 3 - 5 } 2010.06 .01 & 2000 & Top & Middle & Bottom & & 79.06 \\
2010.06 .05 & 201 & 245 & 203 & 5.24 & 79.18 \\
2010.06 .09 & 2000 & 202 & 252 & 203 & 5.21 & 81.51 \\
2010.06 .13 & 2000 & 221 & 264 & 221 & 4.60 & 82.59 \\
2010.06 .17 & 248 & 270 & 245 & 4.32 & 80.35 \\
2010.06 .23 & 2000 & 240 & 270 & 240 & 4.90 & 79.90 \\
2010.07 .03 & 240 & 285 & 252 & 5.02 & 81.58 \\
2010.07 .07 & 2000 & 235 & 240 & 240 & 4.58 & 80.93 \\
2010.07 .12 & 2500 & 240 & 260 & 240 & 4.75 & 81.12 \\
2010.07 .17 & 3500 & 245 & 260 & 240 & 4.70 & 76.69 \\
2010.07 .20 & 2400 & 240 & 280 & 245 & 5.87 & 78.23 \\
\hline
\end{tabular}

the reaction system totally had 6 thermocouples which respectively indicated the temperature of inlet, upper bed, lower bed and outlet. Catalyst was loaded in a $6.4 \mathrm{~m}^{3}$ upper layer and a $6.6 \mathrm{~m}^{3}$ lower layer). Industrial operation started from June, 2012 and has applied from then on. At the start of the operation, because the new device was put into operation for the first time, the bed temperature had risen to a high $838^{\circ} \mathrm{C}$. But we tried our best to control the temperature and the device soon reached full-load operation. 
Table 7. Test data of the $600 \mathrm{~h}$ industrial side-line test with oxygen-containing feed gas.

\begin{tabular}{|c|c|c|c|c|c|c|c|}
\hline \multirow{2}{*}{ Time } & \multirow{2}{*}{ Space velocity, $\mathrm{h}^{-1}$} & \multicolumn{4}{|c|}{ Temperature, ${ }^{\circ} \mathrm{C}$} & \multirow{2}{*}{ Tail gas, CO\% } & \multirow{2}{*}{ Shift ratio, \% } \\
\hline & & Inlet & Top & Middle & Bottom & & \\
\hline 2011.07.20 & 2500 & 216 & 298 & 278 & 245 & 2.97 & 87.87 \\
\hline 2011.07.22 & 2500 & 217 & 304 & 293 & 263 & 3.06 & 87.51 \\
\hline 2011.07.24 & 2500 & 224 & 318 & 300 & 269 & 3.83 & 84.49 \\
\hline 2011.07.26 & 2500 & 225 & 318 & 296 & 273 & 3.38 & 86.25 \\
\hline 2011.07.28 & 3000 & 223 & 324 & 332 & 301 & 4.05 & 83.63 \\
\hline 2011.07.30 & 3000 & 223 & 344 & 346 & 323 & 4.01 & 83.79 \\
\hline 2011.08.01 & 3000 & 224 & 307 & 352 & 336 & 6.81 & 73.19 \\
\hline 2011.08.03 & 3000 & 224 & 304 & 349 & 332 & 5.91 & 76.53 \\
\hline 2011.08.05 & 3000 & 225 & 306 & 355 & 338 & 5.33 & 78.72 \\
\hline 2011.08.07 & 3000 & 221 & 309 & 359 & 338 & 5.15 & 79.40 \\
\hline 2011.08.09 & 3000 & 222 & 318 & 362 & 341 & 4.47 & 82.01 \\
\hline
\end{tabular}

Note: CO\% in feed gas was 31.20\%; Xco = (inlet CO\%-outlet CO\%)/[inlet CO\% $\times(1+$ outletr CO\%)] $\times 100 \%$.

Part of the industrial operation data of QDB-04 in Shandong Lunan Second Nitrogen Fertilizer Plant are shown in Table 8 . It is seen from Table 8.

1) At the beginning of the startup with low operation load, the $216^{\circ} \mathrm{C}-225^{\circ} \mathrm{C}$ inlet temperature was enough to satisfy the requirements for CO outlet content (20\% - 23\%) in the methanol device which showed that the QDB-04 catalyst has good low temperature activity.

2) Due to the serious overheating of the QDB-04 catalyst at the beginning of the startup and several times of startups and shutdowns in the operation, the inlet temperature was improved about $10^{\circ} \mathrm{C}$ (from $255^{\circ} \mathrm{C}$ to $266^{\circ} \mathrm{C}$ at full load) in the latter period of operation. In spite of this, the catalyst can still satisfy the requirements of the methanol device which shows the good activity and structure stability of QDB-04.

3) During the operation, the bed resistance drop maintained almost the same which showed that there're no powdering and crushing of the catalyst in the course of operation and it keeps good strength and strength stability.

4) In the course of the new device running, there're frequent startups and shutdowns and frequent changes of load, pressure, water/gas ratio, but QDB-04 catalyst still maintained stable performance and it showed resistance to operating condition fluctuations.

\subsection{Sample Analysis of the Industrially Used Catalyst}

\subsubsection{Exterior}

During the overhaul of Lunan Chemical Fertilizer Plant in March, 2012, part of the upper layer catalyst in the shift furnace was replaced because there was an overheating to $838^{\circ} \mathrm{C}$ of the upper layer catalyst which might affect the long time production. After comparing the upper and lower layer samples, it was found that QDB-04 catalyst remained its whole particle with no caking and powdering, albeit the upper layer overheated sample got a paler color.

\subsubsection{Strength}

Comparison of the strength between the fresh sample and used sample is listed in Table 9. Table 9 shows that QDB-04 catalyst retained 98\% of its strength in both the upper and lower part of the furnace after one year's operation. The overheated samples still retained $85 \%$ of its strength.

\subsubsection{Bleeding of the Active Components}

Contents of alkaline metal $\mathrm{K}_{2} \mathrm{O}$ and active component $\mathrm{MoO}_{3}$ are examined for the samples from upper and lower parts of the upper layer after one year industrial operation (see Table 10). The data show that their alkaline metal 
Table 8. Part of the operation data of QDB-04.

\begin{tabular}{cccccccccc}
\hline Date & Gas flowrate & \multicolumn{9}{c}{ Temperature, ${ }^{\circ} \mathrm{C}$} \\
& $\mathrm{N} \mathrm{m}^{3} / \mathrm{h}$ & Inlet & \multicolumn{2}{c}{ First layer } & \multicolumn{2}{c}{ Second layer } & Outlet & Outlet & Resistance drop \\
\hline 2012.06 .04 & 5251 & 216 & 399 & 401 & 438 & 429 & 432 & 23.21 & 6 \\
2012.06 .10 & 8303 & 225 & 414 & 418 & 428 & 422 & 424 & 23.17 & 8 \\
2012.06 .25 & 10764 & 221 & 405 & 412 & 418 & 415 & 408 & 18.64 & 10 \\
2012.07 .15 & 12900 & 218 & 399 & 408 & 413 & 414 & 419 & 21.95 & 12 \\
2012.08 .01 & 27871 & 242 & 325 & 398 & 432 & 424 & 430 & 21.38 & 30 \\
2012.09 .25 & 30814 & 255 & 329 & 395 & 425 & 413 & 421 & 20.31 & 30 \\
2012.10 .10 & 32025 & 254 & 335 & 402 & 428 & 420 & 420 & 22.8 & 30 \\
2012.10 .25 & 27885 & 252 & 326 & 393 & 430 & 417 & 427 & 20.9 & 30 \\
2012.11 .30 & 24123 & 244 & 404 & 436 & 440 & 439 & 436 & 22.53 & 23 \\
2012.12 .16 & 33977 & 246 & 318 & 380 & 427 & 404 & 424 & 21.67 & 30 \\
2013.01 .07 & 33100 & 259 & 351 & 414 & 435 & 426 & 430 & 21.45 & 30 \\
2013.01 .21 & 33490 & 266 & 339 & 403 & 432 & 418 & 428 & 22.74 & 30 \\
2013.02 .27 & 33753 & 264 & 357 & 418 & 433 & 440 & 436 & 22.35 & 31 \\
2013.03 .31 & 28962 & 230 & 348 & 417 & 428 & 428 & 427 & 20.2 & 30 \\
2013.04 .21 & 33285 & 234 & 351 & 410 & 419 & 419 & 418 & 19.74 & 30 \\
2013.05 .15 & 33860 & 236 & 349 & 415 & 424 & 425 & 424 & 21.32 & 31 \\
\hline
\end{tabular}

Table 9. Strength comparison of QDB-04 catalyst samples before and after industrial operation.

\begin{tabular}{cccc}
\hline Catalyst & & Strength, N/cm & Retention ratio, \% \\
\hline Fresh sample & & 137.8 & $/$ \\
After one & Upper part of the upper layer & 135.4 & 98.3 \\
\multirow{2}{*}{ Year's operation } & Lower part of the upper layer & 118.4 & 85.9 \\
& Middle part of the lower layer & 136.9 & 99.3 \\
\hline
\end{tabular}

Table 10. Comparison of the active component of QDB-04 catalyst samples before and after industrial operation.

\begin{tabular}{cccc}
\hline Catalyst & & $\mathrm{MoO}_{3}$ content, & \multicolumn{1}{c}{$\mathrm{K}_{2} \mathrm{O}, \%$} \\
\hline Fresh sample & & 7.49 & 8.23 \\
After one & Upper part of the upper layer & 7.36 & 8.24 \\
Year's operation & Lower part of the upper layer & 6.76 & 8.18 \\
\hline
\end{tabular}

content remains the same to that of the fresh sample after one year's operation, so are the $\mathrm{MoO}_{3}$ and $\mathrm{K}_{2} \mathrm{O}$ content from the upper layer, but the sample from overheated part shows paler color, and its $\mathrm{MoO}_{3}$ content is $6.76 \%$ which is obviously lower than that of the upper layer sample because of the sublimation of the active component during the period of overheating at over $800^{\circ} \mathrm{C}[10]$.

\subsubsection{Phase Composition and Pore Structure}

Table 11 shows the comparison of the XRD phase composition and pore structure of QDB-04 catalyst between samples after one year's industrial operation and the fresh sample. It is shown from Table 11 that specific area of the catalyst from different sites of the furnace was all reduced a little, especially the sample from the lower part of the upper layer. There are also bigger pore distribution changes in the lower part samples from the upper 
Table 11. Comparison of the physi-chemical performance of QDB-04 catalyst before and after industrial operation.

\begin{tabular}{|c|c|c|c|c|}
\hline \multirow[t]{2}{*}{ Item } & \multirow[t]{2}{*}{ Fresh sample } & \multicolumn{3}{|c|}{ After one year industrial operation } \\
\hline & & $\begin{array}{l}\text { Upper part of upper layer } \\
\text { lower layer }\end{array}$ & $\begin{array}{l}\text { Lower part of upper } \\
\text { layer }\end{array}$ & Middle part of \\
\hline Specific area & 115.1 & 105.2 & 92.7 & 99.4 \\
\hline Pore volume & 0.3609 & 0.2832 & 0.2953 & 0.3283 \\
\hline Average pore radius, nm & 62.81 & 59.50 & 63.63 & 59.99 \\
\hline Most probable radius, $\mathrm{nm}$ & 3.16 & 2.756 & 3.035 & 2.941 \\
\hline \multicolumn{5}{|l|}{ Pore distribution } \\
\hline$<25 \mathrm{~nm}$ & 57.47 & 57.79 & 57.31 & 56.48 \\
\hline $25-50 \mathrm{~nm}$ & 5.11 & 6.05 & 7.28 & 5.91 \\
\hline $50-150 \mathrm{~nm}$ & 13.95 & 20.38 & 22.61 & 27.11 \\
\hline$>150 \mathrm{~nm}$ & 23.39 & 15.79 & 12.79 & 10.51 \\
\hline \multirow[t]{3}{*}{ XRD phase composition } & $\gamma-\mathrm{Al}_{2} \mathrm{O}_{3}$ & $\gamma-\mathrm{Al}_{2} \mathrm{O}_{3}$ & $\gamma-\mathrm{Al}_{2} \mathrm{O}_{3}$ & $\gamma-\mathrm{Al}_{2} \mathrm{O}_{3}$ \\
\hline & $\mathrm{MgAl}_{2} \mathrm{O}_{4}$ & $\mathrm{MgAl}_{2} \mathrm{O}_{4}$ & $\mathrm{MgAl}_{2} \mathrm{O}_{4}$ & $\mathrm{MgAl}_{2} \mathrm{O}_{4}$ \\
\hline & \multicolumn{4}{|c|}{$\mathrm{K}_{2} \mathrm{SO}_{4}$ (micro amount) } \\
\hline
\end{tabular}

layer, among which small pores $(<25 \mathrm{~nm})$ remain almost the same but 50 - $150 \mathrm{~nm}$ medium-size pores are increased and big pores (>150 nm) are reduced obviously. The specific surface and pore structure changes are all caused by the serious overheating at the beginning of the startup.

It is also seen from Table 11 that even for the sample from the overheated part, its specific area was only decreased by $23.9 \%$ and other physical properties change relatively small. This shows that QDB-04 catalyst not only has good activity stability but also has good structure stability.

Table 11 also lists the phase data of QDB-04 before and after industrial application. It is shown that after one year's operation, the physical composition remains almost the same except the micro $\mathrm{K}_{2} \mathrm{SO}_{4}$ engendering in the upper part which further indicates the good structure stability of the catalyst.

\section{Conclusions}

1) Experiment tests show that QDB-04 is a sulfur tolerant CO shift catalyst with special support and new-type assistant which have the properties of high strength and strength stability, high resistance to powdering, good low-temperature activity and activity stability, low alkaline metal bleeding ratio and easy sulfurization.

2) Industrial application results show that QDB-04 catalyst has low activation temperature, high low-temperature activity, high activity stability and high resistance to low water/gas ratio, which can perfectly satisfy the requirements of the methanol device of Lunan Nitrogen Fertilizer Plant. It overcomes the shortcomings (such as peeling and powdering that cause the rise of bed resistance) of the $\gamma$-Al2O3-based industrial catalyst.

3) Analysis of the unloaded sample shows that QDB-04 catalyst stands the one-year industrial operation and serious over-heating accident and retains the original bed resistance and catalysis activity. The unloaded samples keep high retention ratios of strength, pore structure and active component which show its good strength, strength stability and corrosion resistance. It can satisfy the requirements of methanol process conditions to catalyst activity, strength and structure stability.

\section{References}

[1] Lu, C.R., et al. (1997) Current Situation of Sulfur Tolerant Shift Conversion Catalysts at Home and Abroad. Chemical Fertilizer Industry, 24, 13-17.

[2] Liu, J., et al. (2007) Newly Developed QDB-04 Sulfur Tolerant Catalyst for CO Shift and Its Application. M-Sized Nitrogenous Fertilizer Progress, 1, 53-56.

[3] Li, X., et al. (2000) Studies on MgO-Promoted Sulfur Tolerant Shift Catalyst Carrier. Qilu Petrochemical technology, 28, 14-16. 
[4] Tian, Z.M., et al. (2001) The Effects of Process Condition on Activity of Tolerant-Sulfur Shift Catalyst. Shanghai Chemical Industry, 18, 14-16.

[5] Jin, X.X. (1998) Advances of CO Shift Technology. Small-Scale Nitrogenous Fertilizer, 8, 1-8.

[6] Zong, Q.Y. (2000) Influence of Potassium on the Activity of Sulfur Tolerant Shift Catalyst. Industrial Catalysis, 8, 36-39.

[7] Zong, Q.Y. (2002) Studies on Catalytic Performance of Potassium-Promoted Co-Mo Based Sulfur Tolerant Shift Catalyst. Chinese Journal of Catalysis, 23, 521-524.

[8] Lin, R.C. (2001) The XPS and TPR Characterization of Sulfur-Resisted Catalysts for Water Gas Shift Promoted by $\mathrm{K}_{2} \mathrm{CO}_{3}$. Chemical Journal of Applied Chemistry, 18, 25-28.

[9] Hou, P., Meeker, D. and Wise, H. (1983) Kenetic Studies with a Sulfur Tolerant Water Gas Shift Catalyst. Journal of Catalysis, 80, 280-285. http://dx.doi.org/10.1016/0021-9517(83)90253-1

[10] Xiang, D.H. and Liu, H.Y. (1992) Manual of Chemical Fertilizer Catalyst. Chemical Fertilizer Industry Publishing House, 231. 